\title{
Composite Texture of Chitin and Keratin in an Animal Organ, Lingula seta
}

\author{
Kiichiro TANAKA, Nobuhiko Katsura, Takashi SAKU,* \\ and Shigeo KASUGA ${ }^{* *, \dagger}$ \\ Departments of Oral Biochemistry, *Oral Pathology, and \\ ** Oral Histology, School of Dentistry, \\ Nagasaki University, Nagasaki 852, Japan
}

(Received June 29, 1987)

\begin{abstract}
Lingula unguis seta gave an oriented X-ray diffraction photograph without any artificial treatments, and the seta was a composite texture of chitin and keratin. Chitin columns of $1 \mu \mathrm{m}$ in diameter were embedded in keratin matrix. The crystal structure of the chitin was $\beta$-form, and also that of keratin. The molecular axes of keratin and chitin were parallel to the axis of the seta.

KEY WORDS Lingula / Brachiopod / Seta / Chitin / Keratin / Composite Material / Composite Texture /
\end{abstract}

Composite material of polymers has become of interest in recent years, because composite material has some new properties each component does not have, as a result of blending. ${ }^{1}$ Biopolymers are good model-materials for polymerphysists and polymerchemists. We

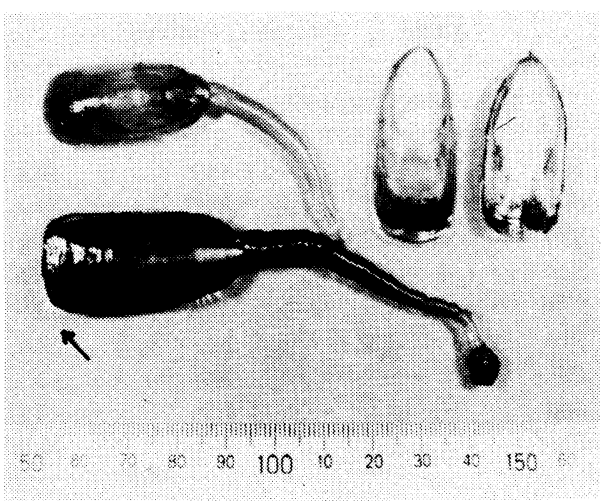

Figure 1. Lingula unguis. The setae are present around a shell as shown by arrow. The shells at the right side of the top are inside out. This portion is calcified with calcium phosphate. found an interesting composite texture in an animal organ, Lingula seta.

Lingula belonging to brachiopod is an interest animal (Figure 1) because of the particular position in the genealogical tree. The species has been living through five thousand million years without any evolution in spite of the changes of biological environment, so it is sometimes called a "living fossil". ${ }^{2}$ The seta of the animal is an important organ for capture of food.

The seta is a needle-like very transparent organ, so we expected it should have a crystal structure. Certainly, it gave a good oriented Xray diffraction photograph, and we found the seta is a composite texture of chitin and keratin.

\section{MATERIALS AND METHODS}

Lingula unguis was obtained commercially at a fish shop in Yanagawa city which is by the

${ }^{\dagger}$ Present address: Central Research Laboratories, Wakunaga Pharm. Co., Ltd. 1624 Shimokotachi Koda-cho Takatagun, Hiroshima 729-64, Japan. 
seaside of the Ariake sea. The seta was about $30-50 \mu \mathrm{m}$ in diameter.

\section{Chemical Analysis}

The setae were hydrolyzed for $16 \mathrm{~h}$ at $110^{\circ} \mathrm{C}$ with $6 \mathrm{~N} \mathrm{HCl}$ for amino acid analysis, and for $7 \mathrm{~h}$ at $100^{\circ} \mathrm{C}$ with $4 \mathrm{~N} \mathrm{HCl}$ for glucosamine analysis. Each hydrolysate was applied to an amino acid analyzer. Neutral sugar and uronic acid were determined by the Anthrone meth$\mathrm{od}^{3}$ and the Carbazole method, ${ }^{4}$ respectively, using the hydrolysate with $4 \mathrm{~N} \mathrm{HCl}$.

\section{$X$-Ray Diffraction}

The setae were washed with water and made up into a bundle for X-ray sample without any other treatments. An X-ray diffraction photograph was taken by using $\mathrm{Ni}$-filtered $\mathrm{Cu}-K_{\alpha}$ radiation in cylindorical technique. The incident beam was perpendicular to the axis of a cylindorical camera. The axis of seta was parallel to the axis of camera (set 1) or perpendicular to it (set 2). In the case of set 2, the seta vibrated 15 degrees to record the higher layer diffractions.

\section{Electron Microscopy}

A seta was embedded into epoxy resin, sliced with a microtome, and stained with uranyl acetate. The slices were observed with an electron microscope in a transmissional system under the magnification of about $2000-10000$.

\section{Immunohistochemistry}

Tissues near the seta were fixed in $95 \%$ ethanol for $12 \mathrm{~h}$ at $4{ }^{\circ} \mathrm{C}$, and routinely processed and embedded in paraffin. Sections were cut in $5 \mu \mathrm{m}$ thickness. For the immunohistochemical staining, an indirect immunoperoxidase method utilizing rabbit anti-keratin antisera and peroxidase conjugated goat antisera against rabbit IgG was employed. The reaction product was developed with 3,3'diaminobenzidine. Nuclear counterstaining was carried out with hematoxylin. As the control, different steps of reactions were omitted and specific antibodies were replaced by normal rabbit sera.

Keratin was extracted from human sole and the antisera was raised in rabbits. The details of the immunochemical procedures in the preparation of antisera to keratin have been described elsewhere. ${ }^{5}$

\section{Purification of Chitin}

Chitin was isolated by Hackmann's method ${ }^{6}$ from the setae. Dried setae of $5 \mathrm{mg}$ were powdered by a mortar and pestle in liquid nitrogen, and suspended in $5 \mathrm{ml}$ of $2 \mathrm{~N} \mathrm{HCl}$ for $48 \mathrm{~h}$ at room temperature, then centrifuged. The precipitate was heated with $5 \mathrm{ml}$ of $1 \mathrm{~N}$ $\mathrm{NaOH}$ at $100^{\circ} \mathrm{C}$ for $12 \mathrm{~h}$. The remaining insoluble material was collected by centrifugation, and washed with water completely and dried. $1.8 \mathrm{mg}$ of chitin were obtained. No amino acid was detected from the chitin.

\section{Infrared Spectrometry}

Five hundred $\mu \mathrm{g}$ of the chitin were made into a tablet with $\mathrm{KBr}$ for infrared spectrometry.

\section{Chitinase Digestion}

The chitin $(1 \mathrm{mg})$ was suspended into $5 \mathrm{ml}$

Table I. Chemical analysis

\begin{tabular}{|c|c|c|c|}
\hline \multicolumn{4}{|c|}{ Amino acid composition in residues $/ 1000$} \\
\hline Hyp & N.D. & Val & 40.8 \\
\hline Asp & 148.9 & Met & 24.8 \\
\hline Thr & 44.7 & Ile & 8.2 \\
\hline Ser & 70.9 & Leu & 31.6 \\
\hline Glu & 87.9 & Tyr & 32.9 \\
\hline Pro & 61.4 & Phe & 28.4 \\
\hline Gly & 169.4 & $\mathrm{His}$ & 82.1 \\
\hline Ala & 69.6 & Lys & 37.8 \\
\hline Cys & 22.5 & Arg & 36.8 \\
\hline \multirow{4}{*}{\multicolumn{2}{|c|}{$\begin{array}{l}\text { Protein } \\
N \text {-Acetylglucosamine* } \\
\text { Uronic acid } \\
\text { Neutral sugar }\end{array}$}} & \multicolumn{2}{|c|}{$40 \%(w / w)$} \\
\hline & & \multicolumn{2}{|c|}{$60 \%(w / w)$} \\
\hline & & \multicolumn{2}{|c|}{ N.D. } \\
\hline & & \multicolumn{2}{|c|}{$1 \%(w / w)$} \\
\hline
\end{tabular}

N.D., not detected.

* determined by content of glucosamine. 
of $50 \mathrm{mM}$ acetate buffer, pH 5.2 with $500 \mu \mathrm{g}$ of chitinase-GODO (EC 3.2.1.14) obtained commercially from Seikagaku-Kogyo Co., Ltd. (Tokyo), and digested at $37^{\circ} \mathrm{C}$ for $36 \mathrm{~h}^{7}$ The amount of released $N$-acetylglucosamine was measured by Morgan-Elson method. ${ }^{8}$

\section{RESULTS AND DISCUSSION}

Table I shows a seta to contain a large amount of glucosamine, and the molar ratio of cystein to be high. The uronic acid was not detected, and the amount of neutral sugar was negligible, so its major components may be

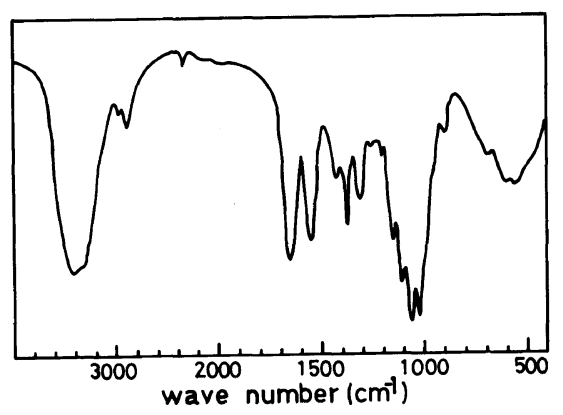

Figure 2. Infrared spectra.

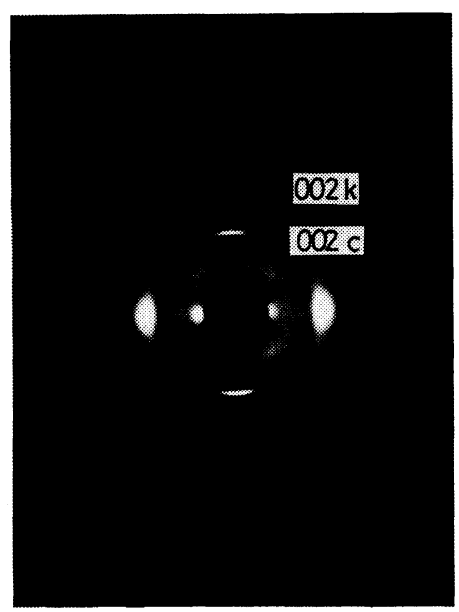

Figure 3. An X-ray diffraction photograph taken in cylindrical technique. The diffraction spots can be indexed by the unit cells of $\beta$-chitin and $\beta$-keratin. $002 \mathrm{k}$, 002 refraction of $\beta$-keratin ( $3.34 \AA$ ); $002 \mathrm{c}, 002$ refraction of $\beta$-chitin $(5.15 \AA)$. The seta is a composite texture. chitin and keratin. The presence of chitin in the seta was confirmed by infrared spectrophotometry and chitinase-digestion test as fol-

Table II. Comparison of observed spacings $\left(d_{\mathrm{o}}\right)$ with calculated spacings $\left(d_{\mathrm{c}}\right)$

Chitin

\begin{tabular}{lllcl}
\hline & $d_{\mathrm{o}}(\AA)$ & Intensity & $d_{\mathrm{c}}(\AA)$ & Index \\
\hline Zero layer & 10.5 & $\mathrm{~s}$ & 10.5 & 010 \\
& $4.7-4.2^{\dagger}$ & $\mathrm{vs} ; \mathrm{d}$ & 4.70 & 100 \\
& & & & 110 \\
& $2.3^{\dagger \dagger}$ & $\mathrm{m} ; \mathrm{d}$ & 2.35 & 200 \\
& & & 2.29 & 140 \\
1st layer & 2.13 & $\mathrm{w}$ & 2.14 & 220 \\
& 7.52 & $\mathrm{~m}$ & 7.34 & 011 \\
2nd layer & 5.32 & $\mathrm{w}$ & 4.27 & 101 \\
& 5.14 & $\mathrm{vs}$ & 5.15 & 002 \\
& 4.61 & $\mathrm{w}$ & 4.62 & 012 \\
3rd layer & 3.47 & $\mathrm{w}$ & 3.47 & 102 \\
& $3.3^{+\dagger \dagger}$ & $\mathrm{vs} ; \mathrm{d}$ & 3.27 & 013 \\
& 2.47 & $\mathrm{~m}$ & 2.45 & 033 \\
& & & 2.45 & 123 \\
& 2.06 & $\mathrm{w}$ & 2.08 & 043 \\
& & & 1.94 & 203 \\
4th layer* & 2.57 & $\mathrm{~m}$ & 2.57 & 004 \\
& 2.2 & $\mathrm{w}$ & 2.31 & 024 \\
& & & 1.94 & 104 \\
\hline
\end{tabular}

$d_{\mathrm{c}}$ was calculated by an orthorhombic cell $a=4.7 \AA$, $b=10.5 \AA$, and $c=10.3 \AA$ (fibre axis).

${ }^{\dagger}$ contains 200 of keratin; ${ }^{\dagger \dagger}$ contains 400 of keratin;

${ }^{+\dagger+}$ contains 002 of keratin.

* recorded by Set 2 (see the text).

Keratin $d_{\mathrm{o}}(\AA) \quad$ Intensity $\begin{array}{ccc}d_{\mathrm{c}}^{*} & d_{\mathrm{c}}^{* *} \\ (\AA) & (\AA)\end{array}$ Index

\begin{tabular}{llllll}
\hline Zero layer & $4.7-4.2^{\dagger}$ & vs; d & 4.73 & 4.63 & 200 \\
& $2.3^{\dagger \dagger}$ & m; d & 2.37 & 2.32 & 400 \\
1st layer & 3.95 & w & 3.86 & 3.82 & 201 \\
& 2.26 & w & 2.23 & 2.20 & 401 \\
2nd layer & $3.3^{\dagger+\dagger}$ & vs; d & 3.34 & 3.34 & 002 \\
& 2.79 & w & 2.73 & 2.71 & 202 \\
4th layer & 1.67 & $\mathrm{~m}$ & 1.67 & 1.67 & 004 \\
\hline
\end{tabular}

* was calculated by a tetragonal cell $a=9.46 \AA, b=6.68 \AA$ (fibre axis).

** was calculated by a monoclinic cell $a=9.57 \AA$, $b=9.99 \AA$, and $c=6.68 \AA$ (fibre axis), $\gamma=103.9^{\circ}$.

${ }^{\dagger}$ contains 100 and 110 of chitin, ${ }^{\dagger \dagger}$ contains 200 and 140 of chitin, ${ }^{\dagger+\dagger}$ contains 013 of chitin.

" recorded by Set 2 .

vs, very strong; s, strong; m, medium; w, weak; d, diffuse. 


\section{K. TANAKA $e t$ al.}

a

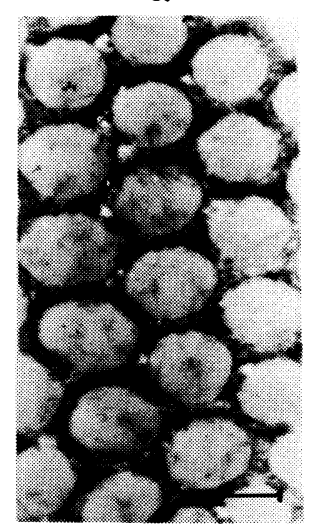

b

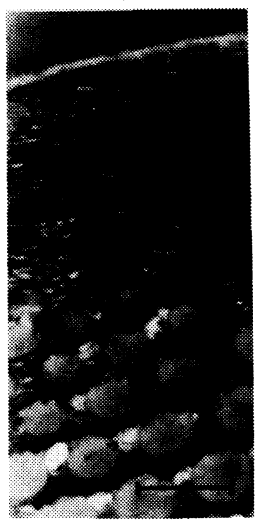

C

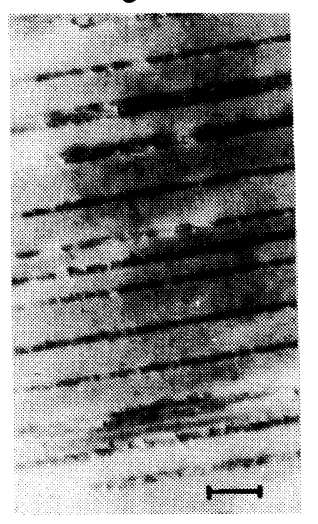

Figure 4. Electronmicrograph of the seta. The specimen was stained with uranyl acetate. Scale bar= $1 \mu \mathrm{m}$. a, cross section, the rods of chitin of about $1 \mu \mathrm{m}$ in diameter are embedded into the matrix of keratin; $b$, cross section of the periphery of seta, the chitin-rods are smaller than these of inside rods, and the fine tentacles present on the surface of seta; $c$, longitudinal section, the rods are oriented in parallel to the axis of seta.

lows. A small amount of insoluble material was observed in the incubation mixture of the chitin and chitinase even after $36 \mathrm{~h}$, but it was less than $0.1 \mathrm{mg}$. The chitin was digested almost completely. The released $N$-acetylglucosamine was $47 \%(2 / \mathrm{w})$. The infrared spectra showed a spectrogram of chitin which contained the absorption spectra attributed to $N$-acetyl group at $1650 \mathrm{~cm}^{-1}$ and $\beta$-D-glucosidic linkage at $895 \mathrm{~cm}^{-1}$ as shown in Figure 2. And there are no absorption bands assigned to sulfate group or carboxyl group. It is evident by these results that a seta contains chitin. Keratin was detected immunohistochemically. X-Ray analysis was done to make clear the secondary structure of them.

An X-ray photograph of the seta is shown in Figure 3 and the index of the reflections in Table II. The reflections could be classified into two kinds assigned to $\beta$-chitin and $\beta$ keratin. Those of $\beta$-chitin could be indexed by an orthorhombic cell of $a=4.7 \AA, b=10.5 \AA$, and $c=10.3 \AA$ (fibre axis) which is completely consistent with the crystal structure reported Dweltz. ${ }^{9}$ As shown in Table II, the reflections of $\beta$-keratin could be indexed by both a tetragonal cell $(a=9.46 \AA$ and $c=6.68 \AA$ (fibre axis))

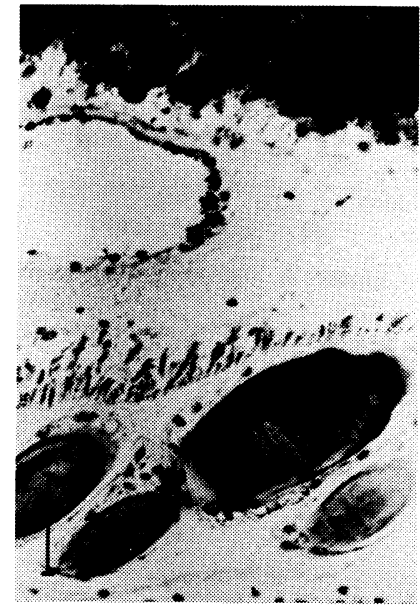

Figure 5. Immunoperoxidase staining of keratin. A nuclear counterstaining, hematoxylin. Scale bar $=$ $50 \mu \mathrm{m}$. Only the outskirt of the columns showed positive reaction for keratin. The control sections showed no positive reactions. Honeycomb structures are demonstrated.

and a monoclinic cell $(a=9.57 \AA, b=9.99 \AA$, and $c=6.68 \AA$ (fibre axis) and $\gamma=103.9^{\circ}$ ) proposed by Fraser and MacRae. ${ }^{10}$ They suggested that $\beta$-keratin takes both crystal structures in bulk material. The molecular chains in the tetragonal cell are parallel with each other, but 
antiparallel in the monoclinic cell, and these two structures are mixed randomly. The observed reflections of $\beta$-keratin of the seta were so few that we were not able to justify the validity of their conclusions at all. The observed reflections could be indexed by their two unit cells.

The electron microscopic photographs show that the columns of about $1 \mu \mathrm{m}$ in diameter (Figures $4 \mathrm{a}, \mathrm{b}$, and $\mathrm{c}$ ), are embedded into the matrixes stained with uranyl acetate. Uranyl acetate stains a diffuse structure around a compact structure. The degree of crystallinity of keratin was lower than that of chitin evidently from X-ray diffraction photograph. Therefore the columns are chitin and the matrix is keratin. This is evident from the immunohistochemical observation more directly (Figure 5). The matrix stained with keratinantibody surrounds the columns like a honeycomb.

We have not investigated the mechanical properties of setae. Chitin is a very rigid material, and keratin is more elastic than chitin. Setae may have both rigidity and elasticity, and these mechanical properties are useful for capture of food.

\section{REFERENCES}

1. T. Chou, R. L. McCullough, and R. B. Pipes, Scientific American, 255(4), 167 (1986).

2. J. R. Richardson, Scientific American, 255(3), 96 (1986).

3. E. W. Yemm and A. J. Willis, Biochem. J., 57, 508 (1954).

4. T. Bitter and H. Muir, Anal. Biochem., 4, 330 (1962).

5. T. Saku, H. Okabe, Y. Yagi, E. Sato, and N. Tsuda, Acta Pathol. Jpn., 34, 1031 (1984).

6. R. H. Hackmann, Aust. J. Biol. Sci., 7, 168 (1954).

7. M. Yabuki, K. Takayama, A. Ando, and T. Fujii, Tech. Bull. Fac. Hort. Chiba Univ., 34, 21 (1984).

8. J. L. Reissig, J. L. Strominger, and L. F. Leloir, J. Biol. Chem., 217, 959 (1955).

9. N. E. Dweltz, Biochim. Biophys. Acta, 51, 283 (1961).

10. R. D. B. Fraser and T. P. MacRae, J. Mol. Biol., 5, 457 (1962). 